\title{
The association between pain, balance, fall, and disability in patients with lumbar spinal stenosis with vascular claudication
}

\author{
Musa Güneş ${ }^{1}$, Tarık Özmen ${ }^{1}$, and Tuğba Moralı Güler ${ }^{2}$ \\ 'Department of Physiotherapy and Rehabilitation, Faculty of Health Sciences, Karabuk University, Karabuk, Turkey \\ ${ }^{2}$ Department of Neurosurgery, Faculty of Medicine, Karabuk University, Karabuk, Turkey
}

Received May 11, 2021

Revised June 29, 2021

Accepted July 9, 2021

Handling Editor: Jin-Woo Shin

\section{Correspondence}

Musa Güneş

Department of Physiotherapy and Rehabilitation, Faculty of Health

Sciences, Karabuk University, Karabuk 78050, Turkey

Tel: +90 (370) 4189078

Fax: +90 (370) 4189353

E-mail: musagunes339@gmail.com

\begin{abstract}
Background: The effect of lumbar spinal stenosis (LSS) and peripheral vascular disease (PVD), which occurs with similar degenerative conditions, when seen together, has not been studied. The aim of this study is to examine and compare the relationship between pain, balance, disability, fear of falling, and kinesiophobia in LSS patients with intermittent vascular claudication (IVC).

Methods: Seventy-two patients diagnosed with LSS using magnetic resonance imaging participated in this study. Thirty-five patients with IVC symptoms and showing vascular lesions by lower extremity venous and arterial Doppler ultrasonography imaging were included in the IVC-LSS group. The pain, static balance, dynamic balance, disability, fear of falling, and kinesiophobia were evaluated using the numeric rating scale, single leg stance test, Time Up and Go (TUG), the Oswestry Disability Index (ODI), Fall Efficacy Scale-International (FES-I), and Tampa Scale for Kinesiophobia (TSK), respectively.

Results: Age and female sex were found to be higher in the IVC-LSS group $(P=0.024$; $P=0.012$ ). The IVC-LSS group had a shorter single leg stance time and TUG test duration, pain intensity, ODI, FES-I, and TSK scores were higher than patients with LSS $(P=0.001)$. Pain, fear of falling, and kinesiophobia were moderately correlated with disability in the IVC-LSS group. No relationship was found between pain and dynamic balance. Also, the pain was not related to kinesiophobia.

Conclusions: The findings indicated that IVC causes loss of balance and an increase in pain, disability, fear of falling, and kinesophobia in patients with LSS.
\end{abstract}

Key Words: Accidental Falls; Catastrophization; Disability Evaluation; Fear; Intermittent Claudication; Low Back Pain; Peripheral Vascular Diseases; Postural Balance; Spinal Stenosis; Ultrasonography, Doppler.

\section{INTRODUCTION}

The primary symptom of lumbar spinal stenosis (LSS), a problem of narrowing of the spinal canal that occurs with aging, is intermittent claudication in the lower extremities [1]. Intermittent vascular claudication (IVC), which can be seen due to venous insufficiency [2] and peripheral arterial diseases (PAD) [3], increases with walking and decreases when resting $[4,5]$. On the other hand, intermittent neurogenic claudication (INC) is aggravated by ambulation and standing, and is relieved by trunk flexion and sitting [6]. The effects of LSS and PAD, which cause intermittent claudication with similar degenerative conditions, can be seen simultaneously [7]. Therefore, LSS and peripheral vascular (c) This is an open-access article distributed under the terms of the Creative Commons Attribution Non-Commercial License (http://creativecommons.org/licenses/by-nc/4.0/), which permits unrestricted non-commercial use, distribution, and reproduction in any medium, provided the original work is properly cited.

(c) The Korean Pain Society, 2021
Author contributions: Musa Güneş: Writing/manuscript preparation; Tarık Özmen: Supervision; Tuğba Moralı Güler: Investigation. 
Table 1. Comparison of vascular versus neurogenic claudication

\begin{tabular}{lll}
\hline \multicolumn{1}{c}{ Outcomes } & \multicolumn{1}{c}{ Vascular } & \multicolumn{1}{c}{ Neurogenic } \\
\hline Pain location & Calves & Back, buttock, thighs \\
Exacerbating factors & Activity, walking & Walking/standing erect, spinal extension \\
Alleviating factors & Rest & Spinal flexion \\
Onset of relief & Immediate & Within minutes \\
Walking uphill & Painful & Painless \\
Imaging & Doppler ultrasonography & Magnetic resonance imaging of lumbar spine \\
\hline
\end{tabular}

diseases (PVD) occuring together in advanced ages should be distinguished from each other [8].

Pain and claudication are primary symptoms of the LSS [9]. Pain affects the daily life activities of individuals and leads to severe disability and a decrease in functional level $[10,11]$. Pain and disability result in kinesiophobia in individuals with LSS [12]. Intermittent claudication [9,13] and problems such as flexed posture, motor deficit, and a decrease in spinal proprioception lead to a decrease in functional mobility and poorer balance $[14,15]$. In previous studies, the loss of balance has been identified in about half of individuals with LSS $[15,16]$.

In IVC, leg pain that is aggravated by walking is more severe than low back pain and reduces the functional capacity of individuals by restricting their mobility and physical activity [6]. Impaired peripheral circulation in individuals with PVD with IVC is associated with sensory and motor nerve dysfunction in the lower extremities [17]. This causes poorer balance function [17-19], and an increase in the risk of falling [20]. It is also known that individuals with IVC have more history of falls and stumbles $[17,18]$. These individuals have a fear of falling as a result of weak physical, social, and psychological functions [21].

Since symptoms in LSS depend on the position of the spine, conservative approaches focus more on the lumbar region [22], while treatment of IVC involves the lower extremities [23]. The vascular problems accompanying LSS should be detected and their effects should be examined. A biopsychosocial approach to pain perception and determination of related factors are necessary for an individualized rehabilitation program [24].

In the literature, it has been reported that loss of balance and the risk of falling, disability, and fear of movement increase in IVC and LSS. However, to the authors' knowledge, there is no study investigating the effects of these factors on individuals with coexisting IVC and LSS (IVCLSS). The author's hypothesis is that IVC may cause more pain, loss of balance, disability, fear of falling, and kinesiophobia in individuals with LSS. It is also thought that there is a relationship between pain, balance, and other psychosocial functions. This study aimed to compare and examine the relationship between pain, balance, disabil- ity, fear of falling, and kinesiophobia in the individuals with LSS with and without IVC.

\section{MATERIALS AND METHODS}

\section{Participants and settings}

This study is a descriptive and cross-sectional study evaluating the IVC status of individuals with LSS who were admitted to the neurosurgery outpatient clinic from September 2020 to January 2021. Seventy-five individuals aged 35-80 years, diagnosed with LSS by magnetic resonance imaging, and able to walk independently participated in the study. The presence of intermittent claudication, factors increasing and decreasing pain, cardiovascular disease, and additional pathologies were questioned in the participants (Table 1) [5].

During the evaluation, one patient with severe pain and two patients with poor communication due to old age were excluded from the study. Of the 72 patients with LSS included in the study, lower extremity bilateral venous and arterial Doppler ultrasonography was requested for further imaging. Individuals who had a vascular lesion in the leg according to imaging results, and whose clinical symptoms met the definition of IVC by a neurosurgeon, were included in the IVC-LSS group as a result of the consultation of the vascular surgeon [25]. Arterial and venous calcification, occlusion, and narrowing ranged from minimum flow disturbances to maximum flow disturbances. No classification was used for rating. Individuals suffering from severe limb ischemia (e.g., gangrenous tissue, ulcerated limb), a neurological disease that may affect balance (hemiplegia, multiple sclerosis, Parkinson, etc.), visual or vestibular system impairment, lower extremity joint disease, or surgical history in the past year, malignancy in the spine, and those with severe lung and cardiovascular disease were excluded. The patients were evaluated after the examination of the neurosurgeon. This study was approved by the Karabuk University ethics committe (2020/349). Written consent was obtained from all patients. 


\section{Data collection instruments}

Demographic and physical characteristics of the patients such as age, sex, weight, height, smoking, chronic diseases, duration of symptoms, pain localization were recorded. Pain intensity was evaluated using the numerical rating scale (NRS) during activity and rest, both back and leg (0$10,10=$ worst imaginable pain) [26].

The static balance of the participants was measured with the single leg stance test (SLST) with eyes open (for a maximum of 30 seconds) [27]. Three tests were allowed on the affected side and the best result was used. The dynamic balance and mobility were assessed by the Time Up and Go (TUG) test. TUG is the measurement of the walking performance of the patient to get up from a standard chair, walk 3 meter, and come back and sit again. The time was recorded in seconds [28].

The disability level of individuals was assessed by the Oswestry Disability Index (ODI). The ODI revised form, developed to evaluate functional disability in low back pain, has 10 items (pain intensity, personal care, lifting, walking, sitting, standing, sleeping, social life, traveling, and changing the degree of pain). Pain-related disability ranges from 0 to 100 points (100 = worst) [29].

Fear of falling was assessed using the Fall Efficacy Scale-International (FES-I). The FES-I is a self-reported questionnaire that evaluates the level of anxiety about falls during activities of daily living. The questionnaire contains 16 items scored on a four-point scale $(1=$ not at all concerned to $4=$ very concerned) providing a total score ranging from 16 (absence of concern) to 64 (extreme concern) [30].

The Tampa Scale for Kinesiophobia (TSK) is a 17 -item scale used to measure individuals' fear of movement/reinjury. All items are rated on a 4-point Likert scale ( $1=$ strongly disagree, $4=$ strongly agree). Four out of 17 items (the 4th, 8th, 12th, and 16th item) are scored inversely ( $1=$ strongly agree, $4=$ strongly disagree). The total sum has a minimum score of 17 (low fear) and a maximum of 68 (high fear) [12].

\section{Sample size}

The sample size was calculated using G*Power 3.1 software (Heinrich-Heine-Universität Düsseldorf, Düsseldorf, Germany). The sample size of the study [31] was calculated with $80 \%$ power, an effect size of 0.68 , and a significance level of $\alpha=0.05$ based on two independent groups and two-way hypothesis. Accordingly, it was found that it was necessary to work with 70 individuals, including at least 35 individuals in both groups.

\section{Statistical analysis}

The IBM SPSS Statistics 21.0 (IBM Co., Armonk, NY) program was used for the statistical analysis of the data. The relevance of data to a normal distribution was evaluated using the Kolmogorov-Smirnov test. Descriptive analyses were presented using mean and standard deviation for the normally distributed variables, and tables of frequencies, median, and minimum and maximum values for the non-normally distributed and ordinal variables. The independent $t$-test and Mann-Whitney $U$-test were used for comparisons between the two groups, and the chi-square test was used to compare qualitative data. Spearman's rank-order correlation analysis was used to determine the correlations between the variables. For the correlation coefficients, $r>0.7$ was considered a strong correlation, those between 0.3 and 0.7 a moderate correlation, and those between 0 and 0.3 a weak correlation [32]. The level of statistical significance was set at $P<0.05$ and $P<0.01$.

\section{RESULTS}

A total of 72 patients with LSS (48 females and 24 males) were included in the study. The demographic characteristics of the 37 LSS and 35 IVC-LSS patients with are shown in Table 2. The mean age of the IVC-LSS group (60.9 \pm 7.9 years $)$ was higher than the LSS group $(56.4 \pm 8.4)(P=$

Table 2. Demographic characteristics of the patients $(n=72)$

\begin{tabular}{lccc}
\hline \multicolumn{1}{c}{ Variable } & IVC-LSS $(\mathrm{n}=35)$ & LSS $(\mathrm{n}=37)$ & $P$ value \\
\hline Age $(\mathrm{yr})$ & $60.9 \pm 7.9$ & $56.4 \pm 8.4$ & $0.024^{*}$ \\
BMI $\left(\mathrm{kg} / \mathrm{m}^{2}\right)$ & $33.8 \pm 4.8$ & $30.9 \pm 4.5$ & $0.012^{*}$ \\
Sex & & & \\
$\quad$ Female & $29(82.9)$ & $19(51.4)$ & $0.006 * *$ \\
$\quad$ Male & $6(17.1)$ & $18(48.6)$ & \\
Smoking & & & \\
$\quad$ Never & $18(51.4)$ & $16(43.2)$ & 0.547 \\
$\quad$ Former & $12(34.3)$ & $12(32.4)$ & \\
$\quad$ Current & $5(14.3)$ & $9(24.3)$ & \\
Diabetes mellitus & $15(42.9)$ & $2(5.4)$ & $0.001 * *$ \\
Hypertension & $23(65.7)$ & $10(27.0)$ & $0.002 * *$ \\
Cardiovascular disease & $13(37.1)$ & $5(13.5)$ & $0.029 *$ \\
Affected leg side & & & \\
$\quad$ Unilateral & $24(68.6)$ & $23(62.2)$ & 0.568 \\
$\quad$ Bilateral & $11(31.4)$ & $14(37.8)$ & \\
Positive history of falls & $24(68.6)$ & $8(21.6)$ & $0.001 * *$ \\
Duration of symptoms (mo) & $48(18-240)$ & $36(3-216)$ & $0.022 *$ \\
\hline
\end{tabular}

Values are presented as mean \pm standard deviation, number (\%), or median (minimum-maximum).

LSS: lumbar spinal stenosis, IVC-LSS: intermitant vascular claudication with lumbar spinal stenosis, BMl: body mass index.

$* P<0.05, * * P<0.01$. 
0.024). In the IVC-LSS group, body mass index, female sex, diabetes, hypertension, and cardiovascular disease were significantly higher than the LSS group $(P=0.012 ; P=0.006$; $P=0.001 ; P=0.002 ; P=0.029)$. A history of smoking, which is known as a risk factor for vascular diseases, was not different between the groups $(P=0.547)$. The IVC-LSS group had higher fall history in the last 6 months $(P<0.001)$ and longer duration painful symptoms $(P=0.022)$.

As shown in Table 3, the IVC-LSS groups had more severe pain. When the groups were compared, single leg stance time was shorter, TUG test time was longer, and ODI, FES-I, and TSK scores were higher in the IVC-LSS group $(P=0.001)$. In the IVC-LSS group, there was a moder-

Table 3. Comparison of outcomes between IVC-LSS and LSS groups $(\mathrm{n}=$ 72)

\begin{tabular}{lccl}
\hline Variable & IVC-LSS $(n=35)$ & LSS $(n=37)$ & $P$ value \\
\hline NRS & & & \\
$\quad$ Activity & $9(7-10)$ & $8(5-10)$ & $0.001^{* *}$ \\
$\quad$ Rest & $3(1-5)$ & $2(0-6)$ & $0.015^{*}$ \\
SLST (sec) & $4.02(1.98-17.42)$ & $12.32(2.68-27.19)$ & $0.001^{* *}$ \\
TUG $(\mathrm{sec})$ & $15.59 \pm 2.24$ & $11.78 \pm 1.53$ & $0.001^{* *}$ \\
ODI & $69.37 \pm 8.01$ & $52.11 \pm 10.45$ & $0.001^{* *}$ \\
FES-I & $36.20 \pm 7.23$ & $27.49 \pm 5.23$ & $0.001^{* *}$ \\
TSK & $50.89 \pm 3.68$ & $45.86 \pm 3.55$ & $0.001^{* *}$ \\
\hline
\end{tabular}

Values are presented as median (minimum-maximum) or mean \pm standard deviation.

LSS: lumbar spinal stenosis, IVC-LSS: intermitant vascular claudication with lumbar spinal stenosis, NRS: numeric rating scale, SLST: single leg stance test, TUG: Time Up and Go, ODI: Oswestry Disability Index, FES-l: Falls Efficacy Scale-International, TSK: Tampa Scale for Kinesiophobia. $* P<0.05, * * P<0.01$. ate correlation between the TUG test and TSK $(\mathrm{r}=0.525, P$ $<0.001)$. No correlation was found between TUG and other tests $(P>0.05)$. A moderate negative correlation was found between SLST and NRS activity $(\mathrm{r}=-0.344, P=0.043)$ and FES-I $(\mathrm{r}=-0.488, P=0.003)$. Also, a moderate positive correlation was found between ODI and NRS activity $(\mathrm{r}=0.481$, $P=0.003)$, FES-I $(\mathrm{r}=0.558, P<0.001)$, and TSK $(\mathrm{r}=0.479, P$ $=0.004)$. Table 4 shows the correlation between NRS, SLST, TUG, ODI, FES-I, and TSK in LSS and IVC-LSS groups.

\section{DISCUSSION}

To the authors' knowledge, the present study is the first examining balance, disability, fear of falling, and kinesophobia in patients with IVC-LSS. Patients with IVC-LSS had more severe pain, disability, and loss of balance compared to patients with LSS. In addition, fear of falling and kinesophobia levels were highly associated with impaired mobility and disability. The results of the combination of vascular and neurogenic intermittent claudication that affect individuals physically and psychologically are not surprising, but the detection of these results is important.

Chronic back pain occurs in LSS as a result of degenerative changes in the spine. INC, which occurs due to mechanical compression or ischemia in the nerve roots, causes pain aggravated by walking in the leg [6]. Studies have shown that individuals with LSS have severe pain in the back and legs [10,11]. In this study, the pain severity of individuals with LSS was found to be higher and consistent with previous studies. IVC is characterized by pain and cramping of muscle groups [23]. The pain was observed in

Table 4. Spearman's rank-order correlation coefficients values between all outcome measures in IVC-LSS and LSS groups $(n=72)$

\begin{tabular}{|c|c|c|c|c|c|c|c|}
\hline \multicolumn{2}{|c|}{ Group } & \multirow[t]{2}{*}{ TUG } & \multirow[t]{2}{*}{ SLST } & \multirow[t]{2}{*}{ NRS activity } & \multirow[t]{2}{*}{ NRS rest } & \multirow[t]{2}{*}{ ODI } & \multirow[t]{2}{*}{ FES-I } \\
\hline IVC-LSS & TUG & & & & & & \\
\hline & SLST & -0.29 & & & & & \\
\hline & NRS activity & 0.10 & $-0.34 *$ & & & & \\
\hline & NRS rest & 0.02 & -0.26 & $0.38 *$ & & & \\
\hline & ODI & 0.16 & -0.31 & $0.48 * *$ & 0.29 & & \\
\hline & FES-I & -0.17 & $-0.49 * *$ & 0.31 & 0.21 & $0.56 * *$ & \\
\hline & TSK & $0.52 * *$ & -0.25 & 0.31 & -0.02 & $0.48 * *$ & 0.18 \\
\hline \multirow[t]{7}{*}{ LSS } & TUG & & & & & & \\
\hline & SLST & $-0.55 * *$ & & & & & \\
\hline & NRS activity & 0.21 & -0.12 & & & & \\
\hline & NRS rest & $0.55 * *$ & $-0.40 *$ & $0.50 * *$ & & & \\
\hline & ODI & $0.64 * *$ & $-0.41 *$ & $0.53 * *$ & $0.65 * *$ & & \\
\hline & FES-I & $0.53 * *$ & $-0.51 * *$ & $0.38 *$ & $0.49 * *$ & $0.67 * *$ & \\
\hline & TSK & $0.39 *$ & -0.23 & $0.49 * *$ & $0.45 * *$ & $0.68 * *$ & $0.62 * *$ \\
\hline
\end{tabular}

LSS: lumbar spinal stenosis, IVC-LSS: intermitant vascular claudication with lumbar spinal stenosis, TUG: Time Up and Go, SLST: single leg stance test, NRS: numerical rating scale, ODI: Oswestry Disability Index, FES-I: Falls Efficacy Scale-International, TSK: Tampa Scale for Kinesiophobia, r: correlation coefficient.

$* P<0.05, * * P<0.01$. 
IVC due to reduced oxygen supply to the muscles in PAD [23] and standing position due to venous insufficiency [2]. Han et al. [7] reported that patients with IVC-LSS had more pain than LSS, but there was no significant difference. On the contrary, Uesugi et al. [8] observed that the pain of patients with LSS more than those with IVC-LSS. The authors attributed this significant difference to the subjective nature of pain and the difficulty of detecting PAD [8]. IVC causes activity-induced cramps and pain in the distal of the lower extremities, especially in the calf region [5].

Pain intensity increases according to the severity of claudication [23]. In this study, the pain severity of patients with IVC-LSS was found to be higher. Compared to previous studies, the high number of women and the variability and level of disease severity may explain the pain in the present study. Pain is the most important factor in decreasing the quality of life of individuals. Therefore, it should be eliminated. Pain causes functional impairment and disability in activities of daily living. Truszczyńska et al. [10] and Fortin et al. [11] observed ODI scores of 49.3 (17.3) and 49.3 (16.7), respectively and reported that individuals with LSS have a severe disability, similar to the results in this study.

IVC leads to disability and reduced functional capacity by restricting mobility and physical activity [33]. Wood et al. [34] showed that patients with INC had more disability compared to patients with IVC, but could not find a significant difference. Also, the level of disability in patients with IVC was found to be higher than asymptomatic individuals [34]. In the present study, the severity of disability in patients with IVC-LSS was found to be higher than LSS. To the authors' knowledge, in addition to the low back pain in individuals with LSS, calf pain especially contributes to disability in the patients with IVC. In addition, the change from minimum to maximum in the severity of disease in the individuals with LSS and PVD in this study may affect functional performance.

Previous studies have shown that individuals with LSS have poor static balance functions in the anteriorposterior direction $[15,16]$. Somatosensory functions as well as decreases in the low back and lower extremity muscle strength can lead to loss of balance [15]. In studies evaluating dynamic balance and mobility, Kim et al. [28] and Fujita et al. [35] measured the TUG test performance as $14.1 \pm 1.8$ and $11.1 \pm 3.7$ seconds, respectively, and the researchers interpreted this result as balance disorders in individuals with LSS, similar to the results of the present study. In PVD, loss of balance occurs due to circulatory and neurological dysfunction in the lower extremities [17]. Lanzarin et al. [19] reported that there was a change in the anterior-posterior stability in the patients with IVC as a result of the functional impairment and loss of motor control of the muscles [19]. It has been suggested that IVC caused insufficiency in static [17] and dynamic balance [18,19]. In the present study, the IVC-LSS group had poor static and dynamic balance performance. Because the problems caused by IVC are more distally located and lead to deficiencies in muscle function and neural interaction, the combination of LSS and vascular pathologies may cause poorer balance control.

Loss of balance results in fear of falling [14,21]. Previous studies have emphasized that there is a fear of falling in both LSS [36] and PVD [21]. It is assumed that individuals' physical abilities decrease due to the fear of falling and they avoid movement by developing various adaptations [37]. In this study, it was found that both groups had a fear of falling, but the level of fear was higher in individuals with IVC-LSS. Female sex, a history of falling, and higher age are known as risk factors for fear of falling [38]. As reported in previous studies [21,36], the high number of women, the presence of a fall history, and higher age may clarify the higher level of fear of falling in the IVC-LSS group in the present study. Fear, which depends on many factors, is based on personal experience and restricts functional activity [21]. Therefore, physiological and psychological risk factors that cause the fear of falling in patients should be determined.

Kinesiophobia, defined as a fear of movement, is known as an extreme fear of physical movements and activities resulting from a painful injury or re-injury [39]. Pain and disability caused by LSS $[12,34]$ and PVD $[34,40]$ increase kinesiophobia. Sharath et al. [40] examined the effects of pain beliefs on physical activity and health, and showed that individuals with IVC have high levels of kinesiophobia. Besides, it has been shown that pain is believed to be caused by walking, and fear avoidance behavior increases as severity of symptoms increases [40]. In this study, kinesiophobia was found to be higher in both groups. Moreover, individuals with IVC-LSS had more fear of movement. It was not easy to include individuals with similar severity of the disorder. It was observed that the symptoms of individuals with LSS and vascular pathology varied from mild pain to severe muscle cramps in the present study. Furthermore, patients with IVC-LSS had more pain. These factors may define the change in kinesiophobia.

This study is the first examining the relationship between functional and psychosocial factors in individuals with IVC-LSS. Pain was associated with static balance and disability in the IVC-LSS group. In contrast to the LSS group, the moderate correlation between pain and static balance shows that pain leads to functional impairment in LSS due to IVC. Contrary to expectations, no relationship was found between mobility and pain in the IVC-LSS group. IVC leads to deficiency in motor and sensory nerve 
functions [17] and impairment in concentric strength due to adaptations in muscle structure [41]. When these findings are taken into account, in this study, changes in muscle and nerve functions in individuals with IVC-LSS may have affected dynamic balance rather than pain. Wood et al. [34] showed that in individuals with intermitant claudication, pain contributed indirectly to kinesiophobia due to mobility difficulties and depression, and there was no direct relationship between pain and kinesiophobia. Similarly, in this study, no relationship was found between pain and kinesiophobia in the IVC-LSS group. Kinesiophobia may be caused by disability, injury, and fear of increased severity of disease with movement, rather than pain. Individuals avoid movement due to pain $[12,40]$, disability $[12,34]$, and poor balance $[17,26]$. Therefore, it is thought that individuals with IVC-LSS develop a slower and more controlled walking pattern. This explains the relationship between kinesiophobia and dynamic balance. Moreover, the level of disability was observed to be associated with kinesiophobia and fear of falling in individuals with IVC-LSS. For this reason, individuals with functional disabilities avoid moving due to the fear of falling. Loss of static balance was also associated with an increase in fear of falling. It is thought that individuals with IVC-LSS can develop various adaptive strategies while standing or during activity.

One of limitations of the present study is that there was an inequality in sex distribution. There were more women participants than men in the IVC group. The possible explanations of the inequality in sex distribution are that stenosis is seen more widely in women [42], IVC increases with age in women [3], and, though the prevalence of IVCLSS is unclear, the number of male patients admitted to the clinic due to pain is less. Besides, the difference in age distribution can be explained by the fact that vascular pathologies accompany LSS as age progresses $[7,8]$. Secondly, the presence of a control group of healthy individuals could strengthen the authors' interpretations. Third, the participants in this study were not grouped according to IVC severity. The differences in severity of vascular problems can affect outcomes. Further studies in which individuals are grouped according to imaging results are needed. Finally, although the balance tests used in this study can be applied clinically, future studies with balance devices are needed for more objective results.

The results of the present study show that in addition to LSS symptoms, IVC causes disability, fear of falling, and kinesophobia as well as impairment in static and dynamic balance control. In individuals with LSS, the presence of IVC should be evaluated and biopsychosocial factors caused by it should be treated.

\section{CONFLICT OF INTEREST}

No potential conflict of interest relevant to this article was reported.

\section{FUNDING}

No funding to declare.

\section{ORCID}

Musa Güneș, https://orcid.org/0000-0001-8532-2575

Tarık Özmen, https://orcid.org/0000-0002-4483-9655

Tuğba Moralı Güler, https://orcid.org/0000-0003-1083-1601

\section{REFERENCES}

1. Watters WC 3rd, Baisden J, Gilbert TJ, Kreiner S, Resnick DK, Bono CM, et al. Degenerative lumbar spinal stenosis: an evidence-based clinical guideline for the diagnosis and treatment of degenerative lumbar spinal stenosis. Spine J 2008; 8: 305-10.

2. Van der Velden SK, Shadid NH, Nelemans PJ, Sommer A. How specific are venous symptoms for diagnosis of chronic venous disease? Phlebology 2014; 29: 580-6.

3. Norgren L, Hiatt WR, Dormandy JA, Nehler MR, Harris KA, Fowkes FG; TASC II Working Group. Inter-society consensus for the management of peripheral arterial disease (TASC II). J Vasc Surg 2007; 45 Suppl S: S5-67.

4. Han MH, Lee DH, Park KS, Lee YS, Kim KT, Sung JK, et al. Risk factors and incidence for peripheral arterial disease in patients with typical lumbar spinal stenosis. Korean J Spine 2014; 11: 183-7.

5. Messiah S, Tharian AR, Candido KD, Knezevic NN. Neurogenic claudication: a review of current understanding and treatment options. Curr Pain Headache Rep 2019; 23: 32.

6. Deer T, Sayed D, Michels J, Josephson Y, Li S, Calodney AK. A review of lumbar spinal stenosis with intermittent neurogenic claudication: disease and diagnosis. Pain Med 2019; 20(Suppl 2): S32-44.

7. Han SH, Jeon CH, Lee DH, Choo HS, Chung NS. Clinical and radiological characteristics of concomitant peripheral arterial obstructive disease in patients with lumbar spinal stenosis. Pain Physician 2013; 16: 513-20.

8. Uesugi K, Sekiguchi M, Kikuchi S, Kanayama M, Takahashi $\mathrm{K}$, Chiba K, et al. Lumbar spinal stenosis associated with peripheral arterial disease: a prospective multicenter observational study. J Orthop Sci 2012; 17: 673-81.

9. Katz JN, Dalgas M, Stucki G, Lipson SJ. Diagnosis of lumbar 
spinal stenosis. Rheum Dis Clin North Am 1994; 20: 471-83.

10. Truszczyńska A, Truszczyński O, Rąpała K, Gmitrzykowska E, Tranowski A. Postural stability disorders in rural patients with lumbar spinal stenosis. Ann Agric Environ Med 2014; 21: 179-82.

11. Fortin M, Lazáry À, Varga PP, Battié MC. Association between paraspinal muscle morphology, clinical symptoms and functional status in patients with lumbar spinal stenosis. Eur Spine J 2017; 26: 2543-51.

12. van Wilgen CP, Stewart R, Patrick Stegeman PT, Coppes M, van Wijhe M. Fear of movement in pre-operative patients with a lumbar stenosis and or herniated disc: factor structure of the Tampa scale for kinesiophobia. Man Ther 2010; 15: 593-8.

13. Tomkins-Lane CC, Battié MC. Predictors of objectively measured walking capacity in people with degenerative lumbar spinal stenosis. J Back Musculoskelet Rehabil 2013; 26: 34552.

14. Leinonen V, Määttä S, Taimela S, Herno A, Kankaanpää M, Partanen J, et al. Impaired lumbar movement perception in association with postural stability and motor- and somatosensory-evoked potentials in lumbar spinal stenosis. Spine (Phila Pa 1976) 2002; 27: 975-83.

15. Iversen MD, Kale MK, Sullivan JT Jr. Pilot case control study of postural sway and balance performance in aging adults with degenerative lumbar spinal stenosis. J Geriatr Phys Ther 2009; 32: 15-21.

16. Truszczyńska A, Drzał-Grabiec J, Trzaskoma Z, Rąpała K, Tarnowski A, Górniak K. A comparative analysis of static balance between patients with lumbar spinal canal stenosis and asymptomatic participants. J Manipulative Physiol Ther 2014; 37: 696-701.

17. Gardner AW, Montgomery PS. Impaired balance and higher prevalence of falls in subjects with intermittent claudication. J Gerontol A Biol Sci Med Sci 2001; 56: M454-8.

18. Gohil RA, Mockford KA, Mazari F, Khan J, Vanicek N, Chetter IC, et al. Balance impairment, physical ability, and its link with disease severity in patients with intermittent claudication. Ann Vasc Surg 2013; 27: 68-74.

19. Lanzarin M, Parizoto P, Santos GM. Analysis of isokinetic muscle function and postural control in individuals with intermittent claudication. Braz J Phys Ther 2016; 20: 48-57.

20. Laghi Pasini F, Pastorelli M, Beermann U, de Candia S, Gallo S, Blardi P, et al. Peripheral neuropathy associated with ischemic vascular disease of the lower limbs. Angiology 1996; 47: 569-77.

21. Lane RA, Mazari F, Mockford KA, Vanicek N, Chetter IC, Coughlin PA. Fear of falling in claudicants and its relationship to physical ability, balance, and quality of life. Vasc Endovascular Surg 2014; 48: 297-304.

22. Lurie J, Tomkins-Lane C. Management of lumbar spinal stenosis. BMJ 2016; 352: h6234.
23. Spannbauer A, Chwała M, Ridan T, Berwecki A, Mika P, Kulik A, et al. Intermittent claudication in physiotherapists' practice. Biomed Res Int 2019; 2019: 2470801.

24. Mühlhauser Y, Vogt L, Niederer D. How and how fast does pain lead to disability? A multilevel mediation analysis on structural, temporal and biopsychosocial pathways in patients with chronic nonspecific low back pain. Musculoskelet Sci Pract 2020; 49: 102199.

25. Lee DC, Heo DH, Cho KS. Concomitant occlusive vascular lesions of legs in patients with degenerative lumbar diseases: do these lesions influence treatment? J Neurol Surg A Cent Eur Neurosurg 2019; 80: 8-14.

26. Thornes E, Robinson HS, Vøllestad NK. Dynamic balance in patients with degenerative lumbar spinal stenosis; a crosssectional study. BMC Musculoskelet Disord 2018; 19: 192.

27. Lin SI, Lin RM. Disability and walking capacity in patients with lumbar spinal stenosis: association with sensorimotor function, balance, and functional performance. J Orthop Sports Phys Ther 2005; 35: 220-6.

28. Kim HJ, Chun HJ, Han CD, Moon SH, Kang KT, Kim HS, et al. The risk assessment of a fall in patients with lumbar spinal stenosis. Spine (Phila Pa 1976) 2011; 36: E588-92.

29. Fairbank JC, Pynsent PB. The Oswestry disability index. Spine (Phila Pa 1976) 2000; 25: 2940-52.

30. Yardley L, Beyer N, Hauer K, Kempen G, Piot-Ziegler C, Todd C. Development and initial validation of the Falls Efficacy Scale-International (FES-I). Age Ageing 2005; 34: 614-9.

31. Ammendolia C, Côté $\mathrm{P}$, Southerst D, Schneider M, Budgell $\mathrm{B}$, Bombardier $\mathrm{C}$, et al. Comprehensive nonsurgical treatment versus self-directed care to improve walking ability in lumbar spinal stenosis: a randomized trial. Arch Phys Med Rehabil 2018; 99: 2408-19.e2.

32. Ratner B. The correlation coefficient: its values range between $+1 /-1$, or do they? J Target Meas Anal Mark 2009; 17: $139-42$.

33. Elnady BM, Saeed A. Peripheral vascular disease: the beneficial effect of exercise in peripheral vascular diseases based on clinical trials. Adv Exp Med Biol 2017; 1000: 173-83.

34. Wood DW, Haig AJ, Yamakawa KS. Fear of movement/(re) injury and activity avoidance in persons with neurogenic versus vascular claudication. Spine J 2012; 12: 292-300.

35. Fujita N, Sakurai A, Miyamoto A, Michikawa T, Otaka Y, Suzuki S, et al. Stride length of elderly patients with lumbar spinal stenosis: multi-center study using the Two-Step test. J Orthop Sci 2019; 24: 787-92.

36. Broscheid KC, Behrendt T, Hamacher D, Böker S, Gagelmann T, Schmidt C, et al. Effect of a multimodal movement intervention in patients with neurogenic claudication based on lumbar spinal stenosis and/or degenerative spondylolisthesis-a pilot study. Front Med (Lausanne) 2020; 7: 540070.

37. Curcio CL, Gomez F, Reyes-Ortiz CA. Activity restriction related to fear of falling among older people in the Colombian 
Andes mountains: are functional or psychosocial risk factors more important? J Aging Health 2009; 21: 460-79.

38. Scheffer AC, Schuurmans MJ, van Dijk N, van der Hooft T, de Rooij SE. Fear of falling: measurement strategy, prevalence, risk factors and consequences among older persons. Age Ageing 2008; 37: 19-24.

39. Kori SH, Miller RP, Todd DD. Kinesiophobia: a new view of chronic pain behaviour. Pain Manag 1990; 3: 35-43.

40. Sharath SE, Kougias P, Barshes NR. The influence of pain- related beliefs on physical activity and health attitudes in patients with claudication: a pilot study. Vasc Med 2017; 22: 378-84.

41. Harwood AE, King S, Totty J, Smith GE, Vanicek N, Chetter IC. A systematic review of muscle morphology and function in intermittent claudication. J Vasc Surg 2017; 66: 1241-57.

42. Lee BH, Moon SH, Suk KS, Kim HS, Yang JH, Lee HM. Lumbar spinal stenosis: pathophysiology and treatment principle: a narrative review. Asian Spine J 2020; 14: 682-93. 